\title{
Pensamento Computacional por meio da Computação Desplugada: Desafios e Possibilidades
}

\author{
Wilk Oliveira1 $^{1}$, Adão Caron Cambraia ${ }^{2}$, Lucas Tadeu Hinterholz ${ }^{3,4}$ \\ ${ }^{1}$ Instituto de Ciências Matemáticas e de Computação (ICMC) - Universidade de São \\ Paulo (USP) - São Carlos, SP - Brasil \\ ${ }^{2}$ Instituto Federal de Educação, Ciência e Tecnologia Farroupilha (IFFar), Santo \\ Augusto, RS - Brasil \\ ${ }^{3}$ Universidade de Caxias do Sul (UCS), Caxias do Sul, RS - Brasil \\ ${ }^{4}$ Escola Municipal de Ensino Fundamental Nossa Senhora da Glória (EMEF-NSG), \\ Sinimbu, RS - Brasil \\ \{wilk.oliveira@usp.br, adao.cambraia@iffar.edu.br, \\ lthinterholz@ucs.br\}
}

\begin{abstract}
In recent years, a series of studies have used Unplugged Computing as a strategy for Teaching Computing in Brazilian Basic Education. Despite the advances, the area still needs to build new trajectories for the production of computer knowledge in basic education. In this sense, the paper presents a position paper aiming to guide the proposition of five challenges, opportunities, and research directions in the Teaching of Computing through Unplugged Computing. The text presents contributions to conduct new research in Unplugged Computing, advancing the literature and maximizing the effects of Unplugged Computing in the teaching of Computing in Brazil.
\end{abstract}

Resumo. Nos últimos anos, uma série de estudos tem usado a Computação Desplugada como uma estratégia para Ensino de Computação na Educação Básica Brasileira. Apesar dos avanços, a área ainda necessita construir novas trajetórias para a produção do conhecimento da Computação na Educação Básica. Nesse sentido, o texto apresenta um artigo de posição a respeito do assunto, a fim de nortear a proposição de cinco desafios, oportunidades e direcionamentos de pesquisa no Ensino de Computação por meio da Computação Desplugada. O texto apresenta contribuições para a condução de novas pesquisas em Computação Desplugada, a fim de avançar a literatura e maximizar os efeitos da Computação Desplugada no ensino de Computação no Brasil.

\section{Introdução}

Desde o ano de 2014, o ensino no Brasil teve seus currículos repensados para os desafios imputados pelo mundo globalizado [Macedo 2014]. Diante disso, o Ministério da Educação (MEC) definiu vinte metas a serem atingidas pela educação através do Plano Nacional de Educação (PNE) e que deverão ser cumpridas até o ano de 2024 [Brasil 2014]. Nesse sentido, passados cinco anos (metade do tempo), é importante analisar quais os avanços conquistados e quais ações ainda faltam construir, 
especialmente no âmbito da Educação Básica (Oliveira, Silva e Hinterholz, 2017; Santana, Oliveira, 2019; Oliveira et al., 2020). Diante disso e da nova Base Nacional Comum Curricular (BNCC), estudos recentes advogam a necessidade da competência conhecida como Pensamento Computacional [Bell et al. 2011], que é definida como a habilidade de resolver problemas a partir do conhecimento de teorias da computação como, por exemplo, a lógica de programação (Santana, Oliveira, 2019; Wing, 2006) e pode ser fomentada por atividades simples de um conceito conhecido por Computação Desplugada [Bell et al. 2011].

A Computação Desplugada sugere atividades de Computação por meios não digitais, por vezes, somente utilizando materiais escolares convencionais [Bell et al. 2011], possibilitando um plano de trabalho sem grande aparato tecnológico e oportunizando um ambiente de desenvolvimento da multidisciplinaridade em tarefas interessantes para o aluno [Bell et al. 2011]. Nos últimos anos, um número crescente de trabalhos envolvendo a Computação Desplugada foram desenvolvidos, no entanto, os resultados ainda precisam ser melhor explorados e as proposições incrementadas (Oliveira et al., 2018; Santos Gama, 2019).

Diante disso, o artigo apresenta um texto de posição (position paper) propondo uma série de desafios, oportunidades e direcionamentos de pesquisa no uso de Computação Desplugada como estratégia de ensino de Computação. Por meio da análise de dois estudos secundários (i.e., mapeamentos sistemáticos) conduzidos por Oliveira et al. (2018) e Santos et al. (2019), identificamos cinco desafios nesse domínio e propomos direcionamentos para cada um desses desafios. Nosso estudo busca contribuir diretamente para reflexão sobre os estudos em Computação Desplugada no Brasil e objetiva abrir caminhos para a condução de novas pesquisas nesse domínio, com intenção de impactar positivamente a Educação em Computação.

\section{Revisão da Literatura}

Esta seção apresenta uma revisão da literatura sobre os principais tópicos abordados nesse trabalho (i.e., Educação em Computação e Computação Desplugada). A comunidade envolvida com Educação em Computação e com discussões sobre o desenvolvimento de um Pensamento Computacional no Brasil tem construído diversos espaços de discussão. Dentre eles, o Workshop sobre Educação em Computação (WEI) e o Simpósio Brasileiro de Educação em Computação (EduComp) são espaços para desenvolver esse debate, pois neles se reúnem professores, pesquisadores e estudantes que atuam em diversos cursos da área: Sistemas de Informação (SI), Engenharia de Software (EGS), Ciência da Computação (CC) e Licenciatura em Computação (LC). Trata-se da constituição de uma comunidade de diálogo e discussões sobre o Pensamento Computacional e o Ensino da Computação ou a constituição de uma comunidade científica de estudos e discussões como forma de potencializar a produção do conhecimento.

Tudo isso tem por objetivo impulsionar pesquisas e práticas que proporcionem que o Pensamento Computacional possa integrar o espaço da escola, proporcionando também que não especialistas acompanhem melhor, por mais tempo e de forma autônoma a produção da ciência e da tecnologia. Embora o conceito de Pensamento Computacional tenha ganhado impulso com o estudo de Wing (2006), essas discussões já perpassavam a educação na década de 1980. No ano de 1986, Seymour Papert, 
professor de Matemática do Massachusetts Institute of Technology (MIT), em seu livro "LOGO: computadores e educação" [Papert 1980] idealizava a programação na Educação Básica. Ao programar com LOGO, uma versão mais primitiva do Scratch, Papert (1980) afirma que a motivação não é a criação de um produto final, mas sim o processo: o erro encontrado pode servir como desafio para construir aprendizagens. Esse movimento também ocorre para o desenvolvimento do Pensamento Computacional por meio da Computação Desplugada.

O Pensamento Computacional, segundo Wing (2006), prioriza conceitos ao invés do ato de programar, o que, significa que com a apropriação desses conceitos, desenvolve-se a capacidade de pensar em múltiplos níveis de abstração e não a mera aplicação de técnicas de programação; gera ideias e não artefatos. Isso significa que o Pensamento Computacional não tem necessariamente como resultado final a produção de software/hardware e reconhece que os conceitos fundamentais da computação estarão presentes para resolver problemas em vários contextos do cotidiano. $\mathrm{O}$ desenvolvimento do Pensamento Computacional contribui para focar no poder de análise, decisão e síntese, na organização e no estabelecimento de prioridades (Silva et al., 2015; Santana, Oliveira, 2019; Valente, 2019), pois não se trata apenas da aplicação desenvolvida com computadores, mas sim de uma forma de raciocínio para a criação e resolução de problemas complexos.

Nesse sentido, é relevante que o Pensamento Computacional integre a Educação Básica e contribua para uma formação integral dos sujeitos. A dupla ruptura epistemológica pretende que os discursos eruditos interajam com os de senso comum, que se "tornem comensuráveis e nessa medida atenuem o desnivelamento que os separa" [Santos 1989]. Uma dificuldade para o desenvolvimento de um Pensamento Computacional na escola é a falta de um espaço para o professor de Computação. Assim, a LC desenvolve iniciativas nas escolas, tais como a prática enquanto Componente Curricular, Programa Institucional de Bolsa de Iniciação à Docência e Estágios Curriculares de forma interdisciplinar para propiciar a incorporação do Pensamento Computacional na Educação Básica [Oliveira et al. 2020].

Essas iniciativas são experiências que contribuem para a formação de professores e para o desenvolvimento de um Pensamento Computacional na Educação Básica [da Cruz et al. 2021]. Trata-se de formas alternativas, tais como gincanas, oficinas, clubes de programação e atividades interdisciplinares que colaboram para gerar uma dinâmica de formação de professores de Computação articulada com o espaço da escola. Um exemplo dessa articulação formativa foi registrado por Cambraia et al. (2016), em que explicitam a concepção de gestor, professor e aluno do curso de LC sobre o planejamento e desenvolvimento de um projeto desenvolvido em um Componente Curricular Articulador, destacando a importância do registro escrito desse processo como forma de alavancar um movimento de permanente reconstrução e vigilância epistemológica para que, ao mesmo tempo em que se valoriza $o$ conhecimento prévio dos alunos, se criem situações de apropriação do conhecimento da Computação.

Não se trata, portanto, de transpor mecanicamente um conhecimento para o outro, mas sim, os saberes escolares "são organizados de forma a atender as finalidades sociais diversas daquelas para as quais foram pensados em seu contexto de produção" [Lopes 2007]. Com isso, na seleção dos conhecimentos escolares não se incluem apenas 
os conhecimentos científicos, mas "todos os saberes que em dado momento histórico são entendidos como válidos e legítimos” [Lopes 2007].

Como discutido por da Cruz et al. (2021), esse processo exige um novo paradigma de formação de professores de Computação, que provoque rupturas com a formação pautada exclusivamente na racionalidade técnica. Assim não existe uma única forma de trabalho para o desenvolvimento do Pensamento Computacional, também não existe um projeto pedagógico único, o que significa que, para as diferentes regionalidades, "há que se forjar diferentes pedagogias, integradas aos conteúdos e aos métodos de cada campo do saber e que considerem as peculiaridades de cada um" [Silva 2007]. Dessa forma, para que o Pensamento Computacional integre o espaço da escola, é necessária uma interlocução dos cursos de formação de professores com escolas de educação básica de forma a integrar e constituir, conjuntamente com as comunidades de pesquisa, uma cidade científica, para articular conhecimentos científicos significativos nas comunidades escolares.

No Curso de LC são desenvolvidas iniciativas para a integração do Pensamento Computacional com conhecimentos pedagógicos e da prática, de forma a proporcionar uma urdidura dos fios que compõem o curso. Não é uma soma de conhecimentos pedagógicos + computacionais + práticas. De acordo com Shulman (1986), o conhecimento específico de cada área é um pressuposto fundamental da profissão docente, porém também é necessário compreender o modo como esse conhecimento se transforma em ensino. Garcia (2005), ao citar uma comunicação de Shulman (1986), afirma que os professores precisam construir pontes entre o significado do conteúdo curricular e a construção desse significado por parte dos alunos. Isso é possível mediante uma compreensão profunda, flexível e aberta do conteúdo, estando também abertos para rever os objetivos, planos e procedimentos mediante interação com os estudantes. De acordo com Wing (2006), o Pensamento Computacional não ocorre apenas com o desenvolvimento de atividades com computadores, as atividades de Computação Desplugada são uma forma de potencializar o desenvolvimento do Pensamento Computacional.

\section{Desafios, Oportunidades e Direcionamentos de Pesquisa}

Esse artigo tem por objetivo apresentar um conjunto de posições (position paper) tratando de desafios relacionados a Computação Desplugada como estratégia para o desenvolvimento do Pensamento Computacional. Os desafios propostos nesse artigo foram baseados nos resultados dos estudos de Santos et al. (2018) e Santos et al. (2019) que realizaram mapeamentos sistemáticos sobre Computação Desplugada no Brasil. Nesse sentido, ambos os estudos previamente citados foram lidos e discutidos por três especialistas em Ensino de Computação, no sentido de identificar e elencar os desafios relacionados ao uso de Computação Desplugada no Brasil. Cinco desafios foram elencados e serão discutidos a seguir.

\subsection{Desenvolvimento de atividades para além da disponibilização de equipamentos}

As políticas públicas para disponibilização de equipamentos nas escolas instrumentalizaram-nas com laboratórios de informática, computadores portáteis, etc. Contudo, de acordo com Alonso et al. (2014), a disponibilidade de equipamentos ainda é um desafio. Primeiro, pela rápida obsolescência dos equipamentos, exigindo atualizações, e, segundo, pela necessidade de periódicas manutenções, que devido ao 
custo acabam ficando para segundo plano e levam os laboratórios de informática a ficarem fechados por falta de manutenção. Diante disso, o desafio da disponibilidade alavanca perspectivas inovadoras em que podemos tornar equipamentos obsoletos em materiais de experiência, em que os próprios alunos os concertem ou explorem novas potencialidades, como reutilização para oficinas de robótica, incorporando $o$ pensamento/raciocínio computacional na escola.

Nesse sentido, é imprescindível seguir fazendo esforços para melhorar esse aspecto. Mas, não é por falta de infraestrutura ideal que se deve imobilizar o processo de ensinar e aprender. Esses desafios abrem portas para iniciativas inovadoras, como o ensino da programação e a Computação Desplugada [Bell et al. 2011], que trata de democratizar o conhecimento da Ciência da Computação por meio do Pensamento Computacional e é temática de diversas pesquisas e estudos acadêmicos vinculados à escola [Farias, Andrade, Alencar 2015]. No caso da programação de computadores, possibilita construir raciocínios diferentes do que, costumeiramente, se exige no Ensino Fundamental ou Médio, onde os conteúdos das exatas são apresentados de forma teoremática (aplicação de fórmulas). A atividade de programação exige um pensamento algorítmico, problemático, em que o aluno aprende a construir as fórmulas e não somente aplicá-las, características presentes no Pensamento Computacional.

Nesse sentido, uma eminente oportunidade é o desenvolvimento de atividades de Computação Desplugada com o desenvolvimento de atividades diversas como destacam Bell et al. (2011) e são proporcionadas apenas com lápis, papel e cola. Então, por meio de construção de tabuleiros e até mesmo utilizando nosso próprio corpo podemos aprender computação sem o uso de equipamentos sofisticados. Além disso, a utilização de equipamentos obsoletos possibilita um trabalho com robótica educacional que incentiva a uma aprendizagem criativa.

\subsection{Desenvolvimento de abordagens para a Educação Infantil}

Apesar de um dos principais interesses da Computação Desplugada ser o estimulo ao Pensamento Computacional a partir da educação infantil [Wing 2006], apenas um dos 15 estudos analisados por Santos et al. (2018) foram conduzidos na educação infantil, representando assim uma desconexão entre os estudos realizados no Brasil e o próprio objetivo geral da Computação Desplugada (que é trabalhar conceitos de Computação ainda na Educação Infantil [Wing 2006]). Além disso, muitas atividades atualmente usadas no contexto da Computação Desplugada não foram propostas e avaliadas com estudantes desse nível educacional (Oliveira et al., 2018; Santos Gama, 2019).

Apesar do desenvolvimento de alguns trabalhos iniciais, como França e Tedesco (2019) que desenvolveram um livro infantil, que narra as aventuras de Lampião Junior e Maria Bonitinha, os quais necessitam resolver problemas do cotidiano com o Pensamento Computacional. Tal situação denota pouca preocupação da comunidade brasileira em relação a educação infantil, afirmando o que já observado em outras áreas [Campos, Füllgraf, Wiggers 2006]. Essa falta de estudos na educação infantil tende a prejudicar a aprendizagem dos estudantes, que acabam não recebendo certos estímulos de grande importância dos primeiros anos da educação formal. Diante disso, destaca-se como desafios a necessidade da realização de estudos em Computação Desplugada que possam ser pensadas para contemplar diretamente a educação infantil.

Diante desse desafio, apresenta-se como oportunidade a condução de estudos que: $i$ ) apliquem e avaliem atividades de Computação Desplugada já existentes no 
contexto da educação infantil; ii) proponham novas atividades de Computação Desplugada focadas nas necessidades e características dos estudantes do ensino fundamental; e iii) desenvolvam materiais didáticos que sejam capazes de prover atividades desplugadas para a educação infantil, bem como, guiar os professores na aplicação dessas atividades em sala de aula.

\subsection{Desenvolvimento e avaliação de novos materiais didáticos}

Como mostrado nos estudos secundários analisados, a maioria (por exemplo, nove de 15 estudos analisados no caso do estudo de Santos et al. (2018), usaram atividades do livro "Computer Science Unplugged: An enrichment and extension programme for primaryaged students" ou de sua versão em Português "Ciência da Computação Desplugada: Ensinando Ciência da Computação sem o uso do computador" [Bell et al. 2011]. Além disso, apenas três dos 15 estudos analisados por Santos et al. (2018) apresentaram propostas de atividades autorais para ensino de Computação por meio da Computação Desplugada.

Tal situação deixa claro que, em geral, há pouca criação de novos materiais didáticos. Desse modo, um dos desafios eminentes da Computação Desplugada no Brasil é o desenvolvimento de novos materiais didáticos (com novas atividades), que possam agregar ao ensino do Pensamento Computacional em diferentes situações e contextos.

Diante disso, destaca-se como uma grande oportunidade da área, o planejamento, desenvolvimento e avaliação de novas atividades usando a técnica da Computação Desplugada, incluindo atividades isoladas para grupos específicos de pessoas (e.g., deficientes visuais, auditivos e físicos), diferentes níveis educacionais (e.g., educação infantil, jovens e adultos). O desenvolvimento dessas atividades pode estar atrelado a produção de livros didáticos pedagógicos destinados ao ensino de Computação.

\subsection{Desenvolvimento de estudos experimentais}

Nos últimos anos a condução de estudos experimentais tem sido um desafio em diferentes nichos de pesquisa e em diferentes áreas de conhecimento. Além disso, no que concerne aos estudos em Computação Desplugada, os resultados dos estudos secundários anteriormente apresentados (Oliveira et al., 2018; Santos Gama, 2019), deixam claro que poucos estudos conduzidos no Brasil aplicando a Computação Desplugada no contexto educacional conduziram alguma experimentação, sendo a maioria, relatos de experiencia com observação do próprio autor em grupos de poucos estudantes [Oliveira et al., 2018].

Diante disso, poucos resultados são encontrados nas experiências com Educação Básica e que mostrem que o desenvolvimento do Pensamento Computacional resulta em um movimento de intensificar as aprendizagens. Tal resultado chama a atenção para a condução de estudos que possam trazer a comunidade resultados mais profundos e que possam ser generalizados e replicados em diferentes contextos. O desenvolvimento desse tipo de estudo, poderá de fato, servir como base sólida para o desenvolvimento de novos estudos que avancem o estado da arte e o estado da prática atual, bem como, possivelmente, trazer resultados estatísticos, que possam comprovar a eficácia (ou não) das abordagens envolvendo Computação Desplugada. 
Diante disso, apresenta-se como oportunidade de pesquisa, a condução de projetos que conduzam experimentações (preferencialmente longitudinais) com análise estatística de dados com turmas de diferentes níveis educacionais, envolvendo atividades de Computação Desplugada já existentes, bem como, de novas atividades que venham a ser propostas. A condução desse tipo de estudo, pode trazer novas descobertas e panoramas para as pesquisas em Computação Desplugada no Brasil.

\subsection{Computação Desplugada e Pensamento Computacional no Ensino Hibrido ou Remoto}

O ensino híbrido já vem sendo discutido como alternativa ao ensino tradicional (BACICH et al. 2015). De modo geral, o ensino híbrido é uma metodologia que combina a aprendizagem presencial e remota, permitindo ao aluno ter parte de sua formação em sala de aula, e parte em ambiente remoto, por exemplo, no lar com sua família (SCHIEHL \& GASPARINI 2016, 2017; BACICH 2016). Nos estudos conduzidos por Santos et al. (2018) e Santos et al. (2019), não foram encontrados estudos envolvendo Computação Desplugada no ensino hibrido ou remoto. Além disso, no ano de 2020, enquanto diversos pesquisadores ainda discutiam sobre como implantar o ensino hibrido, o Brasil sentiu essa necessidade (ou em alguns casos totalmente remoto), em função da pandemia da COVID-19 (Arruda 2020).

Diante dessa necessidade, o ensino de computação também sofreu as consequências, tendo aulas suspensas ou trocadas para o contexto remoto, alterando a forma natural de ocorrência das aulas (Silveira et al. 2020; Rodrigues et al. 2021). Nesse caso, o uso de Computação Desplugada como estratégia para o Ensino de Computação se tornou mais complexa, pois em geral, demanda atividades em grupo, com o uso de objetos físicos "desconectados", por conseguinte, incluindo um novo obstáculo que precisa ser pensando e resolvido.

Sendo assim, um novo desafio surge, o de repensar como a Computação Desplugada pode ser usada no contexto de ensino hibrido ou remoto como estratégia para o Ensino de Computação. Diante disso, advogamos que estudos sejam conduzidos i) adaptando atividades já existentes de Computação Desplugada para o contexto híbrido ou remoto, ii) propondo novas atividades de Computação Desplugada que possam ser facilmente usadas por professores e estudantes em contexto hibrido ou remoto e iii) prover recomendações práticas para professores implantarem Computação Desplugada no ensino hibrido ou remoto. Tais iniciativas podem ser valiosas para incentivar e melhorar o ensino de Computação durante e depois do isolamento causado pela pandemia da COVID-19.

\section{Discussões}

Nesse artigo, apresentamos uma série de desafios e oportunidades relacionadas ao uso da Computação Desplugada como estratégia para o ensino de computação (especialmente na educação básica). De modo geral, os desafios chamam atenção para o desenvolvimento de atividades que não demandem a necessidade de equipamentos tecnológicos. No Brasil, grande parte das escolas, não disponibilizam equipamentos eletrônicos ou estão obseletos e sem uso. Esse desafio não é novo, podendo ser solucionado em duas direções: a) de um lado, os estudantes podem trazer seus equipamentos de casa (celulares, notebooks, etc.), o que também não é uma realidade no 
Brasil e, ou do outro lado, proporcionar atividades de Computação Desplugada para que o conhecimento da ciência da computação possa fluir na escola.

O outro desafio é desenvolvimento de abordagens que favoreçam o ensino de computação na educação infantil. Alguns trabalhos, como explicado no texto já foram desenvolvidos, mas entendemos que mais pesquisadores precisam assumir esse compromisso elaborar iniciativas para o desenvolvimento do Pensamento Computacional na educação infantil, proporcionando acesso a esse conhecimento para todos. Para que isso ocorra, um impulso inicial precisa ser dado, principalmente, por pesquisadores provenientes de cursos de Licenciatura de Computação, que estudam Pedagogia e Computação, potencializando a criação de materiais didáticos que atendem os objetivos das comunidades. A presença do professor de computação proporciona que em cada instituição esse movimento ocorra de diversas formas (criação de cartilhas, de sites educacionais, de aplicativos, jogos, etc.). Potencializando, inclusive a criação de atividades desplugadas para serem trabalhadas no ensino remoto.

Além disso, o desenvolvimento de estudos experimentais que analisem como as crianças aprendem Pensamento Computacional e como esse processo influencia na aprendizagem como um todo e o desenvolvimento de abordagens para o ensino híbrido e remoto foram apresentados. Tais desafios denotam que a área tem crescido nos últimos anos, no enquanto que ainda é necessário pensar novos aspectos relacionados ao uso de Computação Desplugada como estratégia para o ensino de computação. Por meio dos desafios propostos, esperamos contribuir com a comunidade, mas também apresentando caminhos para o uso da Computação Desplugada no Ensino de Computação.

\section{Considerações Finais}

Nos últimos anos, pesquisas envolvendo Computação Desplugada foram conduzidas levando a identificação de diversos potenciais desafios que têm surgido ao longo do tempo. Diante disso, com base nos resultados de estudos secundários sobre Computação Desplugada no Brasil, esse artigo propôs um conjunto de desafios, oportunidades e direcionamentos de pesquisa relacionados a esse tópico. Destaca-se como trabalhos futuros, a importância de uma discussão continua entre pesquisadores da área para promoção de novos desafios, bem como, apresentação de propostas para enfrentar os desafios apresentados nesse artigo.

\section{Referencias}

Arruda, E. P. (2020). Educação remota emergencial: elementos para políticas públicas na educação brasileira em tempos de Covid-19. EmRede-Revista de Educação a Distância, 7(1), 257-275.

Bacich, L. (2016). Ensino Híbrido: Proposta de formação de professores para uso integrado das tecnologias digitais nas ações de ensino e aprendizagem. In Anais do Workshop de Informática na escola (Vol. 22, No. 1, p. 679).

Bacich, L., Neto, A. T., \& de Mello Trevisani, F. (2015). Ensino híbrido: personalização e tecnologia na educação. Penso Editora.

Bell, T., Witten, I. H., Fellows, M., Adams, R.; McKenzie, J. (2011). Ensinando Ciência da Computação sem o uso do computador. Computer Science Unplugged ORG (2011). 
Brasil. (2014) Plano Nacional de Educação-PNE. De 25 de junho de 2014 Lei n. ${ }^{\circ}$ 13.005. 2014. Aprova o Plano Nacional de Educação-PNE e dá outras providências. Diário Oficial da União [da] República Federativa do Brasil (2014).

Cambraia, A. C., Benvenutti, L.M.P.; Moraes, M.G. (2016). O Projeto Integrador num Curso de Licenciatura: a reconstrução de saberes através do movimento dialógico. Hames, C., Zanon, L., Pansera-de-Araújo, MC Currículo Integrado, Educação e Trabalho: saberes e fazeres em interlocução. Ijuí: Ed. UNIJUÍ (2016).

Campos, M. M., Füllgraf, J.; Wiggers, V. (2006). A qualidade da educação infantil brasileira: alguns resultados de pesquisa. Cadernos de pesquisa 36, 127 (2006), 87128.

da Cruz, M. E. K., Marques, S. G., \& Oliveira, W. (2021). Desenvolvimento e Avaliação de Material Didático Desplugado para o Ensino de Computação na Educação Básica. Revista Brasileira de Informática na Educação, 29, 160-187.

Farias, A.; Andrade, W.; Alencar, R. (2015). Pensamento Computacional em sala de aula: Desafios, possibilidades e a formaçao docente. In Anais dos Workshops do Congresso Brasileiro de Informática na Educação, Vol. 4. 1226.

França, R. S.; Tedesco, P. C. A. R. (2019) Sertão.Bit: Um livro-jogo de difusão do Pensamento Computacional. In: V Workshop de Ensino em Pensamento Computacional, Algoritmos e Programação, 2019, Brasília. Anais dos Workshops do VIII Congresso Brasileiro de Informática na Educação.

Garcia, C. M. (2005). Formação de professores: para uma mudança educativa. Porto Editora Porto.

Lopes, A. C. (2007). Currículo e epistemologia. Unijuí Ijuí.

Macedo, E. (2014). Base Nacional Curricular Comum: novas formas de sociabilidade produzindo sentidos para educação. Revista E-curriculum 12, 3 (2014), 1530-1555.

Oliveira, W., Silva, F. C.; Hinterholz, L. T.; Isotani, S.; Bittencourt, I. I. (2018). Computação Desplugada: Um Mapeamento Sistemático da Literatura Nacional. RENOTE 16, 2 (2018).

Oliveira, W.; França, R.; Lemos, A.; Cruz, M. K. da, Scaico, P.; Amaral H.; Teixeira, L. P. (2020). Os Desafios Enfrentados pela Licenciatura em Computação que a Comunidade de Educação em Computação Precisa Conhecer. In Anais do XXVIII Workshop sobre Educação em Computação. SBC.

Oliveira, W.; Hinterholz, L.; Silva, C. (2017) Licenciatura em computação: Desafios e oportunidades na perspectiva do professor. In Anais do Workshop de Informática na Escola, Vol. 23. 705 .

Papert, S. (1980) Logo: computadores e educação. Brasiliense.

Rodrigues, L., Toda, A. M., Oliveira, W., Palomino, P. T., Avila-Santos, A. P., \& Isotani, S. (2021). Gamification Works, but How and to Whom? An Experimental Study in the Context of Programming Lessons. In Proceedings of the 52nd ACM Technical Symposium on Computer Science Education (pp. 184-190). 
Santana, S. J.; Oliveira, W. (2019) Desenvolvendo o Pensamento Computacional no Ensino Fundamental com o uso do Scratch. In Anais do Workshop de Informática na Escola, Vol. 25. 158.

Santos, A.; Gama, R.; Farias, C. (2019) Computação Desplugada no ensino da Computação no Brasil: um mapeamento sistemático da literatura. In Anais da XIX Escola Regional de Computação Bahia, Alagoas e Sergipe. SBC, 565-574.

Santos, B. De S. 1989. Introdução a uma ciência pós-moderna.

Schiehl, E. P., \& Gasparini, I. (2016). Contribuições do Google Sala de Aula para o ensino híbrido. RENOTE-Revista Novas Tecnologias na Educação, 14(2).

Schiehl, E. P., \& Gasparini, I. (2017). Modelos de ensino híbrido: Um mapeamento sistemático da literatura. In Brazilian Symposium on Computers in Education (Simpósio Brasileiro de Informática na Educação-SBIE) (Vol. 28, No. 1, p. 1).

Shulman, L. S. (1986) Those who understand: Knowledge growth in teaching. Educational researcher 15, 2 (1986), 4-14.

Silva, S. F.; Ferreira, A.; Souza, A. A.; Galdino, E., Oliveira, M. L. S.; Neto, S.; Oliveira, W. (2015) Relato de experiência de ensino de computaçao no ensino fundamental em estágio supervisionado da universidade de pernambuco no campus garanhuns. In $23^{\circ}$ Workshop sobre Educação em Computação. 1-10.

Silveira, S. R., Bertolini, C., Parreira, F. J., da Cunha, G. B., \& Bigolin, N. M. (2020). O Papel dos licenciados em computação no apoio ao ensino remoto em tempos de isolamento social devido à pandemia da COVID-19. Série Educar-Volume 40 Prática Docente, 35 .

Valente, J, A. (2019) Pensamento Computacional, Letramento Computacional ou Competência Digital? Novos desafios da educação. Revista Educação e Cultura Contemporânea 16, 43 (2019), 147-168.

Wing, J. M. (2006) Computational thinking. Commun. ACM 49, 3 (2006), 33-35. 